\title{
Polarized Wiggler for NSLS X-ray Ring*
}

\author{
A. Friedman, X. Zhang, S. Krinsky, E.B. Blum and K. Halbach \\ National Synchrotron Light Source Brookhaven National Laboratory Upton, NY 11973
}

\section{Abstract}

We examine the properties of an elliptically polarized wiggler that will generate circularly polarized photons with energy spectrum of 3-12 KeV. The vertical wiggler magnetic field is produced by permanent magnets while the horizontal wiggler field is generated by electric coils capable of $\mathrm{AC}$ excitation. The radiation parameters of the wiggler are presented. Numerical values are calculated for radiation from the wiggler. A conceptual design for such a wiggler is discussed. We consider $A C$ excitation of the wiggler to produce the time modulation of the elliptic polarization. The power dissipated in the vacuum chamber due to the eddy current is considered.

\section{Introduction}

By using circularly polarized X-rays, magnetic properties can be detected much more effectively than with linearly polarized or unpolarized photons. Some effects can only be detected with circularly polarized photons. A typical experiment using circularly polarized light involves measuring the absorption or scattering of the photons by a target. Measurements are taken for right and left circular polarization. The results are then subtracted from each other in order to yield the net polarization. In the example of a magnetic Compton scattering experiment involving a soft magnetic material [1], an axial magnetic field is applied to the target and the scattered photon intensity $I^{+}$is measured, the magnetic field is then reversed and the scattered photon intensity $I^{-}$is measured at the same angle. The net spin of the material can be calculated from:

$$
\frac{I^{+}-I^{-}}{I^{+}+I^{-}}=\frac{f^{+}-f^{-}}{f^{+}+f^{-}} \frac{n_{\uparrow}-n_{\downarrow}}{n_{\uparrow}+n_{\downarrow}} \frac{\Phi_{s p i n}}{\Phi_{0}} .
$$

where $f^{+}, f^{-}$are the fluxes of the right polarized and the left polarized incoming photons respectively, $\Phi_{0}$ is the spin independent scattering cross section, and $\Phi_{\text {spin }}$ is the spin dependent component of the scattering cross section. $f^{+}+f^{-}$is the total photon flux, $n_{\uparrow}+n_{\downarrow}$ is known, $\Phi_{0}$ and $\Phi_{\text {spin }}$ are calculated theoretically. Thus, either the net spin $n_{\uparrow}-n_{\downarrow}$ or the circular polarization paraneter $\left(P_{c} \equiv\left(f^{+}-f^{-}\right) /\left(f^{+}+f^{-}\right)\right)$can be determined from Eq. 1 .

IIard ferromagnetic materials do not yield the same net spin when the DC magnetic field is reversed. It is, thus, beneficial to be able to reverse, instead, the helicity of

\footnotetext{
*Work performed under the auspices of the US Department of Energy under contract no. DE-ACO2-76CH00016.

${ }^{\dagger}$ Lawrence Berkeley Laboratory, Berkeley CA 95720
}

the photon beam. Such reversal is also described by the relations of Eq. 1.

In most cases $\Phi_{\text {spin }} / \Phi_{0} \ll 1$. Hence, there is a serious detection problem. This problem may be alleviated by using an incoming photon beam in which the polarization is modulated sinusoidally in time. The signal detected in this case (Eq. 1) has a fundamental harmonic oscillating with the polarization. The measurement of the intensities $I^{ \pm}$is a small $\mathrm{AC}$ signal (spin effect) modulating a large DC offset (charge effect.). This AC signal can be readily detected using such techniques as lock-in amplifiers and high-pass filters. Fig. 1 describes an elliptically polarized wiggler $[2,3]$ constructed from a vertical permanent magnet wiggler and a horizontal wiggler which may be constructed either of a permanent magnet $[2,3]$ or an electromagnetic magnet [4]. In the case of an electromagnetic wiggler, the oscillation of $P_{c}$ is achieved by exciting the horizontal wiggler with $\mathrm{AC}$ current.

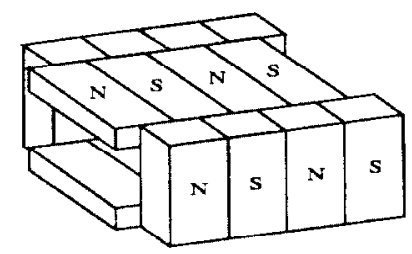

Figure 1

Schematic of an elliptically polarized wiggler

\section{Elliptically polarized wiggler}

Consider a wiggler magnet constructed from a vertical wiggler and a horizontal wiggler superimposed with a $\pi / 2$ phase difference between them [2,3] (Fig. 1). The magnetic field on axis is:

$$
B_{y}=-\frac{2 \pi m c}{e} \frac{K_{y}}{\lambda_{w}} \sin k_{w} z, \quad B_{x}=-\frac{2 \pi m c K_{x}}{e} \frac{\cos k_{w} z}{\lambda_{w}}
$$

where $\lambda_{w}$ is the wiggler period, $k_{w} \equiv 2 \pi / \lambda_{w}$ and $K_{x}, K_{y}$ are the horizontal and vertical wiggler strength parameters. The horizontal wiggler field is weaker than the vertical wiggler field $\left(K_{x} / K_{y}<1\right)$.

In order to get a rough estimate of the properties of the radiation, we utilize the "Bend Source" approximation [2]. The radius of curvature of an electron in an elliptical wiggler is:

$$
\rho=\lambda_{u}\left(1+\xi^{2}\right)^{3 / 2} \frac{\gamma}{2 \pi \sqrt{K_{y}^{2}-\gamma^{2} \xi^{2}}} .
$$


where $\xi$ is the projection of the angle between the wiggler axis and the target of the radiation into the $x-z$ plane. The vertical angles between the viewer and the ares are:

$$
\psi_{+}=\psi+\frac{K_{x}}{\gamma} \quad, \quad \psi_{-}=\psi-\frac{K_{x}}{\gamma} .
$$

where $\psi=\pi / 2-\xi$. Figs. 2 show the photon flux and polarization that can be produced by elliptical wigglers in NSLS $\mathrm{X}$-ray ring straight sections $\left(\epsilon_{y}=2 \times 10^{-9} \mathrm{~m}, \beta_{y}=30 \mathrm{~cm}\right.$, $\left.\sigma_{y^{\prime}}=80 \mu \mathrm{rad}\right)$. The fluxes are computed per wiggler period and should be multiplied by the total number of periods in the wiggler. There is a trade off between the polarization and the flux on axis. A higher $K_{x}$ value will yield a higher degree of circular polarization, but will decrease the photon flux on axis significantly. This is due to the fact that for large $K_{x}$ the angle between the two bends in a period becomes larger, thus producing less radiation on axis. Fig. 2c shows the circular polarization as a function of the electron beam vertical angular spread. Note that a vertical emittance of $\epsilon_{y}=0.2 \times 10^{-9} \mathrm{~m}$ has recently been achieved [5] in the ring, corresponding to an angular spread of 25 $\mu$ radians, which for $K_{x}=1$ will yield $P_{c}>85 \%$.

\section{Conceptual designs for elliptical wigglers}

We consider the vertical wiggler to be built from permanent magnets. Its realization is straightforward since the vertical gap is small and the desired value of $K_{y}=13$ is easily achieved in a configuration with static horizontal field. An electromagnetic horizontal wiggler is of interest, so that the polarization can be modulated. In addition, if it is possible to move the horizontal wiggler relative 10 the vertical wiggler, we could also provide a configuration yielding a tinte-dependent linear polarization.

With an $\mathrm{AC}$ horizontal wiggler there may be eddy currents in the permanent magnct material. To solve this problem, one could use a high resistivity permanent magnet, such as epoxy bound materials. Alternatively it could be possible to build the permanent magnet blocks from laminations. At present, we favor laminated $\mathrm{NdFeB}$ because of its higher remmant field.

The problem of eddy currents may be alleviated by using $0.5 \mathrm{~mm}$ thick iron laminations. The use of transformer iron which contains silicon, would further reduce the generation of eddy currents. A typical configuration of an iron core electromagnetic wiggler is shown in Fig. 3. In reference [6] Halbach developed an algorithm for optimizing such a configuration. Table 1 shows calculation of the various parameters for a wiggler with $\lambda_{w}=16 \mathrm{~cm}$. and $D_{1} / \lambda_{w}=$ 0.2875 . We limited the field in the iron to $B \leq 1$ Tesla in order to use transformer iron, and the current density to $200 \mathrm{Amp} / \mathrm{cm}^{2}$ in order to use air cooled coils. Two gap sizes are examined. The first row in Table 1 represents the field on axis with $J=200 \mathrm{Amp} / \mathrm{cm}^{2}$. The second row represents the current density required for $K_{x}=1$.

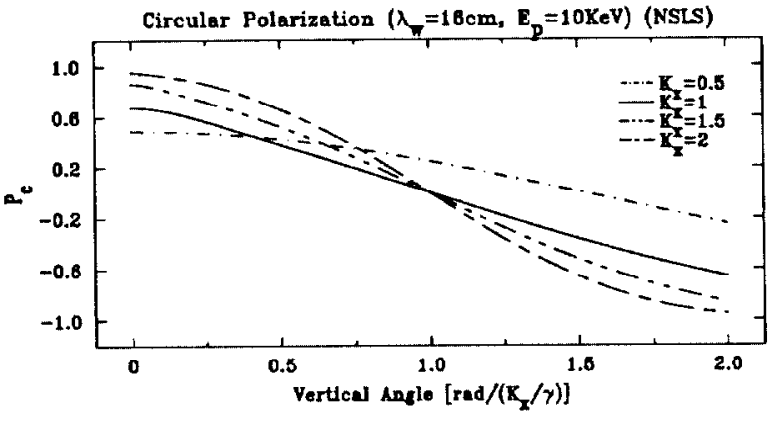

(a)

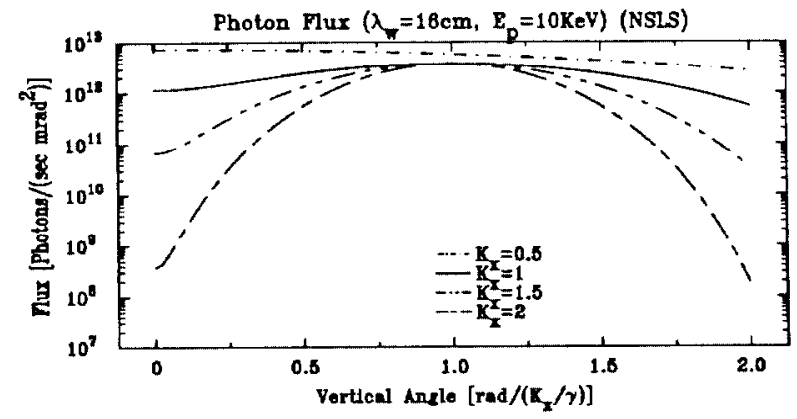

(b)

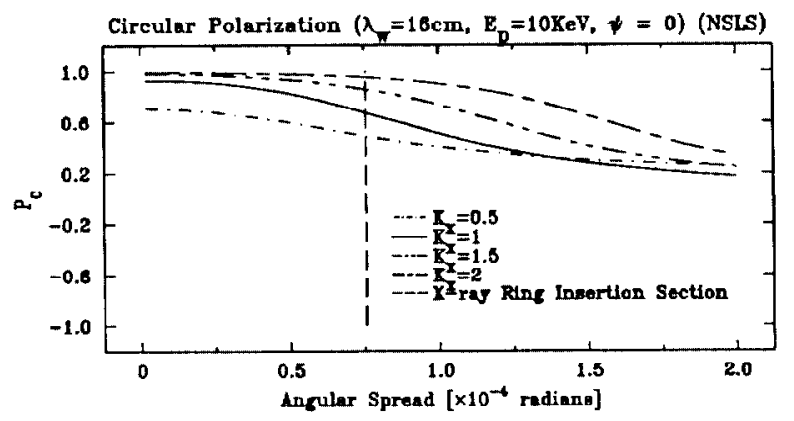

(c)

Figure 2

Elliptical wiggler radiation parameters (a) Circular polarization, (b) photon flux, (c) circular polarization as a function of electron beam vertical angular spread. $\left[K_{y}=13, I=200 \mathrm{~mA}, \Delta \lambda / \lambda=10^{-3}\right]$.

Table 1: Iron Core Electromagnetic Wiggler Parameters $\left(\lambda_{v}=16 \mathrm{~cm}, D_{1}=4.6 \mathrm{~cm}, D_{2}=9.8 \mathrm{~cm}\right)$

\begin{tabular}{|c|c|c|}
\hline & $g=6 \mathrm{~cm}$ & $g=8 \mathrm{~cm}$ \\
\hline $\begin{array}{c}B_{0_{\max }}[\text { Tesla }] \\
\left(J=200 A \mathrm{mp} / \mathrm{cm}^{2}\right)\end{array}$ & 0.1532 & 0.1214 \\
\hline$J\left[\Lambda \mathrm{mp} / \mathrm{cm}^{2}\right]$ & 87.32 & 110.2 \\
\hline
\end{tabular}


The design with $g=8 \mathrm{~cm}$ requires a current density of $110 \mathrm{Amp} / \mathrm{cm}^{2}$ which is well under the limit for air cooled coil.

\section{Eddy currents in the vacuum chamber}

The time variation of the horizontal magnetic field $B_{x}$ gives rise to eddy currents flowing along the vacuum chamber in the axial $z$-direction. Approximating the wiggler magnetic field by a spatially constant dipole field, the eddy current density becomes

$$
J_{z}=-\sigma \dot{B}_{x} y,
$$

where $\sigma$ is the chamber conductivity. The power dissipated is

$$
P=\frac{1}{2 \sigma} \int d v J_{z}^{2},
$$

where the integral is over the chamber wall. Assiming a stainless steel chamber with $\sigma=10^{6} \Omega^{-1} \mathrm{~m}^{-1}$, of thickness $t=1 \mathrm{~mm}$ and length $L$, having elliptical cross-section with half-width $a=1 \mathrm{~cm}$ in the (vertical) $y$-direction and $b=3.5 \mathrm{~cm}$ in the (horizontal) $x$-direction, we find, mks units,

$$
P=\frac{\pi}{2} \sigma t L a^{2} b \dot{B}_{\mathfrak{x}}^{2} F\left(\frac{1}{2},-\frac{1}{2} ; 2 ; \frac{b^{2}-a^{2}}{b^{2}}\right) .
$$

The hypergeometric function has the values $F=1$ for $a=b$ and $F=8 / 3 \pi$ for $a^{2} \ll b^{2}$. Taking $\dot{B}_{x}=$ $2 \pi(100 \mathrm{~Hz})(0.07 \mathrm{I}) / \sqrt{2}$ (the $\sqrt{2}$ factor is due to the sinusoidal behavior of $B_{x}$ in the $z$ direction) we find $P / L=4.5$ watts $/ \mathrm{m}$. A more accurate calculation taking into account the actual field with period $\lambda_{w}=16 \mathrm{~cm}$ yields a power dissipation of 1 watt/period (i.e. 6.4 watts/m). The larger value is due to the increase in the wiggler field as $\cosh k_{w} x$ away from the $y-z$ plane.
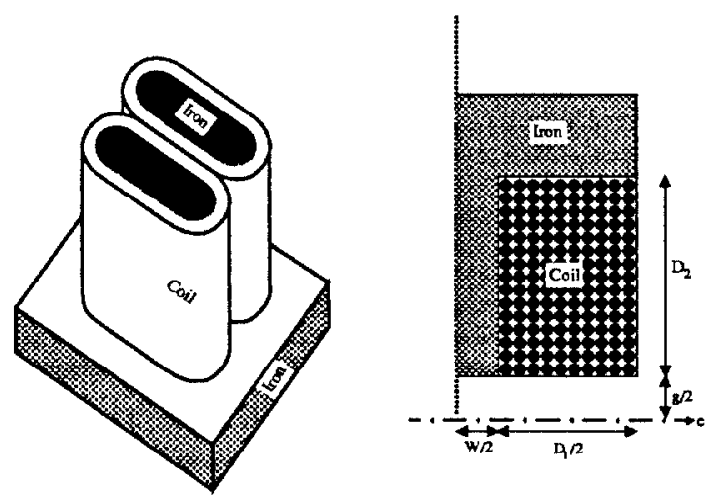

Figure 3

Iron core magnet configuration.

\section{Concluding remarks}

From the results presented, use of an elliptically polarized wiggler looks like a viable way to achieve $3-12 \mathrm{KeV}$ circularly polarized X-rays from the NSLS X-ray ring. Other concepts, such as cross field undulator [7] or a bending magnet will either not yield the high photon energy required or will not produce enough photon flux. These approaches are more sensitive to the electron beam angular spread and hence they are not appropriate for the NSLS ring, which has low vertical $\beta$ in its straight section. In order to increase the sensitivity of detection, AC modulation of the polarization is recommended. This can be done in an elliptical wiggler by constructing one of the wigglers from electromagnets and operating it with $\mathrm{AC}$ current.

A major challenge will be to maintain stability of the orbit with AC excitation of the horizontal wiggler. Time varying correction coils at the entrance and exit of the wiggler will be required, operating at $100 \mathrm{~Hz}$ both in phase and $90^{\circ}$ out of phase with the main wiggler current. Nonlinear effects in the iron can result in time harmonics which might also require correction. Two beam position monitors outside the wiggler separated by $90^{\circ}$ in betatron phase, could be used to determine the needed corrector strengths. The ends of the wiggler magnet should be designed to maint ain satisfactory orbit stability inside the wiggler [8].

A more detailed discussion of some of the issues in this paper can be found in ref [9].

\section{Acknowledgment}

We wish to thank M. Blume, D. Gibbs, J. Hastings, K.J. Kim, D. McWhan and P. Siddons for helpful discussion.

\section{RefERENCES}

[1] N. Sakai, O. Terashime and H. Sekizawa, Nucl. Instrum. and Methods, Phys. Res. Sect. B 221, 419 (1984).

[2] S. Yamamoto, T. Shioga, S. Sasaki and H. Kitamora, Rev. Sci. Instrum. 60 (7), 1834 (1989).

[3] S. Yamamoto, H. Kawata, H. Kitamora and M. Ando, PRL 62 (23), 2672 (1989).

[4] C. Poloni, R. Brocco, B. Diviacco, R.P. Walker and D. Zangrando, Proc. of the 1991 PAC.

[5] J. Safranek and S. Krinsky, in this proceedings.

[6] K. Halbach, NIM A250, 115 (1986).

[7] K.J. Kim, AIP conference proceedings 185, 565 (1987).

[8] K. Halbach, NIM A250, 95 (1986).

[9] A. Friedman, S. Krinsky and F. Blum, BNI report No. 47317, March 1992. 